\title{
Perspective: Does Realism Improve Presence in VR? Suggesting a Model and Metric for VR Experience Evaluation
}

\author{
Sungchul Jung * and Robert. W Lindeman
}

Human Interface Technology Lab (HIT Lab NZ), College of Engineering, University of Canterbury, Christchurch, New Zealand

The concepts of "immersion" and "presence" have been considered as staple metrics for evaluating the quality of virtual reality experiences for more than five decades, even as the concepts themselves have evolved in terms of both technical and psychological aspects. To enhance the user's experience, studies have investigated the impact of different visual, auditory, and haptic stimuli in various contexts to mainly explore the concepts of "plausibility illusion" and "place illusion". Previous research has sometimes shown a positive correlation between increased realism and an increase in presence,

OPEN ACCESS

Edited by: Amela Sadagic, Naval Postgraduate School, United States

Reviewed by: Richard Skarbez,

La Trobe University, Australia Aryabrata Basu,

Emory University, United States

*Correspondence: Sungchul Jung sungchul.jung@canterbury.ac.nz

Specialty section:

This article was submitted to Virtual Reality and Human Behaviour, a section of the journal Frontiers in Virtual Reality

Received: 10 April 2021 Accepted: 30 June 2021 Published: 15 July 2021

Citation:

Jung S and Lindeman RW (2021) Perspective: Does Realism Improve Presence in VR? Suggesting a Model and Metric for VR

Experience Evaluation.

Front. Virtual Real. 2:693327. doi: 10.3389/frvir.2021.693327 but not always, and thus, very little of the work around the topic of presence reports an unequivocal correlation. Indeed, one might classify the overall findings within the field around presence as "messy". Better (or more) visual, auditory, or haptic cues, or increased agency, may lead to increased realism, but not necessarily increased presence, and may well depend on the application context. Rich visual and audio cues in concert contribute significantly to both realism and presence, but the addition of tactile cues, gesture input support, or a combination of these might improve realism, but not necessarily presence. In this paper, we review previous research and suggest a possible theory to better define the relationship between increases in sensory-based realism and presence, and thus help VR researchers create more effective experiences.

Keywords: realism, presence, immersion, evaluation model, metric, illusion, theory, Coherence

\section{INTRODUCTION}

"It's so real!" "This is such a realistic experience!" We believe almost all Virtual Reality (VR) researchers and developers have heard these expressions at least once when successfully delivering immersive VR experience to the general public. Similarly, responses such as "It seems like I'm in another place", or "This looks like my body" are often expressed by the users. General users might not care about the academic distinctions between immersion and illusion, and might even seem confused by them. But clearly, these kinds of responses both implicitly or explicitly indicate the general quality of the VR experience, in a positive direction. Under current circumstances, the most frequently used term is probably related to realism (or realness), and thus using the phrase "the level of realism" might be the easiest way to make the general public understand the quality of a VR experience. On the other hand, in academia, more precise terms, such as immersion (tele-, co-, etc.), presence, embodiment, and body-ownership have been 
suggested to more-precisely define and comprehend how to evaluate the quality of VR experiences (Meehan et al., 2002; Bowman and McMahan, 2007; Slater, 2009; Kilteni et al., 2015; Skarbez et al., 2017).

The two most common high-level concepts, immersion, and place illusion, have been studied along defined lines of objective and subjective aspects, respectively. Immersion is often defined as an objective property Slater (1999), Bowman and McMahan (2007) of a VR system's profile. For example, the visual stimuli from the screen size, resolution, stereo, field-of-view, headtracked head-mounted display (HMD) with full real-time motion capture are critical to simulate a computer-generated experience to the user. Researchers concluded that a system that provides rich virtual surroundings, along with the user's own body movements such as looking around or reaching out to touch a certain object, provides a higher level of immersion than a system that does not support such visual dynamics along with the user's movement. Thus, a VR experience that uses a HMD has higher immersion than a screen-based VR experience. In line with this perspective, realness can be evaluated by, for example, the visual representation of the computer-generated world in terms of the number of triangles and the resolution of textures McDonnell et al. (2012), Latoschik et al. (2017), and enhanced auditory feedback such as spatialized audio (Naef et al., 2002). In addition, supporting the expected additional sensory channels in line with the context of the given VR experience, such as tactile, olfactory, and taste feedback, can increase the sense of realness (Feng et al., 2015; Feng et al., 2016; Jung et al., 2020; Jung et al., 2021a; Jung et al., 2021b).

In contrast, illusion is related to how humans subjectively perceive an immersive experiences: illusion is regarded in terms of psychological aspects (Slater, 2009). To evaluate the quality of these subjective aspects of VR, researchers developed the concepts of presence and body ownership based on the perception or cognition of objects, whether regarding the surroundings or their own avatar body representation, using questionnaire-based constructs or physiological signals (Slater, 1999; Meehan et al., 2002; Slater et al., 2010; Latoschik et al., 2017). The sense of body ownership is regarded as a clear construct, and so at a high level, it may influence the quality of VR experiences. Thus, researchers generally see a positive relationship between it and presence. However, there is still a vague gap in the nature of this relationship, related to the various definitions and understandings of presence. To help address this confusion, two subsequent concepts, place illusion and plausibility illusion, have been suggested (Slater, 2009; Skarbez, 2016). Along with these methods for evaluating the subjective quality of VR experiences, deciding how different levels of immersion might correspond to different levels of illusion has been a focus, measuring how much subjects' responses to events in the virtual world matched reactions to those in the real world (Slater, 2009; Skarbez et al., 2017). In other words, the perception or cognition of the surroundings and their own virtual body are correlated, and thus highly influence the sense of presence and body ownership, leading to subjectively highly-rated qualified VR experiences.
Using these terms and definitions as lenses, revisiting the two opening user comments, "It is so real!" and "This is such a realistic experience!", might be interesting. How can we interpret them? Should we handle them as part of immersion or as illusion comments? Does improved realism also enhance the sense of illusion? In the light of our perspective, a deeper understanding of the term "Realness" or "Realism" is required, since those terms can be situated in both the immersion or illusion aspects, depending on the definition. Realism could be handled as an immersion component if we define it as the extent and quality of the sensory channels. For example, multisensory stimuli improve VR systems; that is, they help provide additional sensory information, such as tactile, olfactory, or taste, which should theoretically lead to a more-immersive VR system compared to traditional VR systems that typically provide only visual and audio cues. In 1999, based on earlier work by Hinckley et al. (1994), Lindeman et al. (1999) introduced the concept of Passive Haptics, mainly related to the use of hand-held props, and observed enhanced performance for manipulating interface tools in virtual environments. Similarly, Insko (2001) designed a study to measure the sense of presence when passive haptics were used. Participants were asked to stand on a $5 \mathrm{~cm}$ physical ledge while they stood on a $20 \mathrm{~m}$ pit in a VR room. The physical ledge allowed users to leverage the feeling of passive haptics, and the researchers found a significant increase in sense of presence. In addition to visual and audio feedback, the use of highlycongruent haptic feedback showed positive impact on the sense of presence.

In 1999, Dinh et al. (1999) investigated the impact of multisensory VR experiences, using a large number of sensory modalities including tactile, olfactory, audio, and visual cues. They found that increasing the number of modalities of sensory input in a virtual environment can increase the sense of presence. However, they reported that increased visual realism did not. After 2 decades, Jung et al. designed a system to deliver additional sensory feedback (vibration, wind, and olfactory cues) in multiple studies (Jung et al., 2020; Jung et al., 2021a; Jung et al., 2021b). In each of these cases, systems with multisensory cues can be seen as (objectively) more immersive. However, if we consider realism within some contextual fidelity, it could be interpreted as an illusionary component.

In this short article, we propose a research question and then present our perspective on this question based on previous research and our own experiences.

Note: This article is based on the assumption that VR has at least visual stimuli, regardless existence of other external sensory stimulation (Skarbez et al., 2021), following the definition of Milgram and Kishino's reality-virtuality continuum (Milgram and Kishino, 1994).

\section{LITERATURE REVIEW FOR IMMERSION AND ILLUSION}

The terms presence and immersion have been suggested and used actively in the VR community or even by the general public sometimes, as representative of the quality of a VR experience, or 
of the expected outcome of exposure to a VR experience. Depending on the technical and psychological context, as well as aspects of the VR experience, many interrelated terms have been used, such as presence, co-presence, tele-presence, social presence, embodiment, and body ownership. Our goal in this section is to revisit the most important terms, while introducing two other terms, Coherence and Realism, that have not been studied as deeply, but that we feel are important. Finally, we suggest a possible model to show the correlation between them in the following next section.

\subsection{Immersion}

Witmer and Singer state that immersion as a subjectively perceived psychological characteristic to the surrounding environment and events in the computer-generated world (Witmer and Singer, 1998). However, and Bowman and McMahan argue that immersion is a systemically objective characteristic of a VR system (Slater, 2009; Bowman and McMahan, 2007). Similarly, Lombard et al. categorized immersion into perceptual immersion and psychological immersion (Lombard et al., 2000). Due to these seeminglycontradictory perspectives of this single term, a clear single point of reference is definitely required in the community. In this article, we support Slater's perspective, that immersion refers to an objective characteristic of a VR system, and address illusion in 2.3.

Our definition of immersion is objective and has clear metrics: more is better. Wide and high-resolution field-of-view (FOV) HMDs provide more immersion than screen-based VR. Also, wide and accurate six-degree-of-freedom (DOF) tracking provides more immersion than three DOF tracking. Similarly, spatial audio cues are more immersion than binaural or monaural audio cues. In light of this perspective, adding more sensory channels should, theoretically, raise the level of immersion. For example, extending secondary feedback cues, such as floor or wearable vibration, wind, and olfactory stimuli that match the visual and audio stimuli, provides more immersion than a system with visual and audio only (Feng et al., 2015; Feng et al., 2016; Jung et al., 2020; Jung et al., 2021a; Jung et al., 2021b).

\subsection{Coherence}

Skarbez et al. first defined the term Coherence to mean the set of reasonable circumstances that can lead to a convincing context without additional explanation, based on a Bayesian prior (Samad et al., 2015; Skarbez, 2016). Coherence is the quality of internal logic and behavioral consistency of a VR experience; thus, the notion does not depend on the faithful representation of realworld experiences (Skarbez et al., 2017). For example, supernatural abilities such as teleportation, invisibility, or flying experienced in the context of science-fiction or fantasy would be regarded as coherent behavior.

\subsubsection{Realism}

Realism or fidelity can be described in as the extent to which the virtual environment emulates the real world (Alexander et al., 2005). Because of the interchangeable usage of the two terms, we use Realism in this article. Similarly, Stoffregen et al. observed that highly realistic systems can produce sensory stimuli that are identical to real-world stimuli, and thus stimulus fidelity can be regarded as an objective characteristic of a simulation. Riccio et al. described experiential fidelity as a subjective experience while action fidelity is a systemic performance (Riccio, 1995). Based on these definitions, conceptualized Coherence as a superset of Realism (Skarbez et al., 2017). Thus, considering the given aspects of the correlation between Realism and Coherence, it is still an open question as to whether Coherence can be regarded as an objective construct or not. As a possible solution for handling Coherence as an objective measure, Skarbez et al. (2017) redefined the domain of the construct "as the set of objectively reasonable circumstances that can be demonstrated by the scenario without introducing objectively unreasonable circumstances." This redefinition is aligned with the previously suggested notion of Experiential Fidelity proposed by Beckhaus and Lindeman, 2011).

\subsubsection{Constructs of Coherence}

Alexander et al. suggest that Realism (or Fidelity) has three subcategories: physical simulation, functional simulation, and psychological fidelity (Alexander et al., 2005). Physical simulation refers to the operational environment such as fully multisensory-enabled experience including visual, aural, tactile, olfaction, and taste stimuli. For example, (Feng et al., 2015; Feng et al., 2016) and (Jung et al., 2020; Jung et al., 2021a; Jung et al., 2021b) achieved a highly-realistic VR experience by using multisensory-cue-enabled VR platforms. Functional simulation is the fidelity of the behavioral representation by the operational equipment in reacting to the tasks executed by the user. While these two components are related to the stimuli from the system, the third one, psychological fidelity, is related to the faithfulness of the psychological effects that the simulation creates with regard to those that would be experienced in a real-world version of the experience.

\subsection{Illusion}

Based on our adoption of Slater's definition of immersion, we categorize Witmer and Singer's description of Illusion as the subjective perception of the psychological characteristic of the surrounding environment and events in the VR environment. In this article, we use Illusion as an equivalent term for Presence that can represent the overall quality of the virtual experience subjectively. For example, Presence is most commonly defined as the feeling of "being there" in a virtual place (Witmer and Singer, 1998). While Presence refers to the feeling of the surrounding environment and events to the first-person's egocentric experience, this notion can be extended to the perception or cognition of other entities' existence, and we call this Co-presence. The concept generally is defined as "being there together" (Schroeder, 2005). Specifically, Goffman et al. stated that Co-presence is related to the sense of mutual perception between two or more (Goffman, 1963). Social-presence also has been suggested to mean the feeling of awareness of being present with other entities with the degree of attention level (Nowak and Biocca, 2003). In this article, we follow and define Social-presence as the moment-by-moment awareness of the Co-presence of 
another sentient being accompanied by a sense of engagement with them Skarbez et al. (2017), and thus we would like to coin a simple representation, "Being there, engaged together," to encapsulate social-presence.

\subsubsection{Constructs of Illusion}

As constructs of Illusion, two logically orthogonal aspects, Place Illusion (PI) and Plausibility Illusion (Psi), have been suggested by (Slater, 2009). He defines PI as "the illusion of being in a place in spite of the sure knowledge that you are not there," and Psi as "the illusion that what is apparently happening is really happening even though you know for sure that it is not". In short, according to this definition, PI can be mapped to the conventional notion of the spatial Presence, while the Psi indicates ones belief that the events happening are things one is actually experiencing (Skarbez et al., 2017).

Embodiment, a sense of "having a body", has been suggested to be a mostly subjective feeling in psychology (Kilteni et al., 2015). Based on the definition of embodiment, virtual body ownership, the sense of feeling "ownership of the given virtual body (of part or whole)" has been developed and researched through numerous studies Banakou et al. (2013), and is regarded as a critical metric for VR experiences, with an implicit agreement on the existence of a correlation with Presence (Yuan and Steed, 2010). To enhance the sense of body ownership in VR, visuo-motor, visuo-tactile, anatomical plausibility Kilteni et al. (2015), and personalized avatar appearance, regardless of whether given directly or indirectly, have been suggested as critical components (Jung and Hughes, 2016; Jung et al., 2017; Jung et al., 2018; Waltemate et al., 2018).

\section{POSSIBLE CORRELATION MODEL FOR IMMERSION AND ILLUSION}

Following Slater's approach for evaluating the quality of VR experience seems to suggest a clear differentiation between Immersion and Illusion (or Presence). However, researchers still observe and report some non-orthogonality between these two high-level concepts. This might be caused due by 1) ambiguity of sub-component definitions, and 2) a possibility of the existence of other factors. For example, Realism can be interpreted either as influenced by either immersion or illusion, depending on the VR context. While the term Realism has been used by both the general public and academia, a clear definition of Realism in VR has not been given. Alternatively, Fidelity is regarded as a similar concept, and Alexander et al. describes Fidelity as the extent to which the virtual environment emulates the real world Alexander et al. (2005) in terms of functional, physical, and psychological perspectives. On the other hand, Stoffregen et al. focus on the sensory stimuli provided by the system (Stoffregen et al., 2003). We believe that Alexander's definition includes Stoffregen's definition, and so prefer Alexander's viewpoint regarding Realism as have done (Skarbez et al., 2017).

Meanwhile, suggest a revised version of a reality continuum, and propose a three-dimensional model that includes "Extent of World Knowledge," "Immersion," and "Coherence." A deep

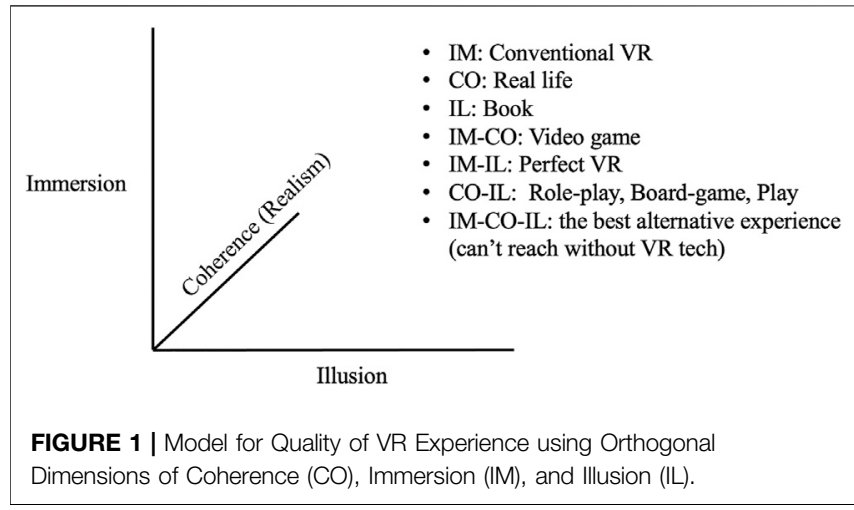

discussion of this important work is beyond the scope of this paper; please refer to it for more details (Skarbez et al., 2021). Noticeable attributes of this model are that Coherence is similar to Psi, and both suggest the importance of the context of the given VR experience. However, Coherence might not be limited to a psychological context. Following Skarbez's description of the term Coherence (Skarbez et al., 2017), we accept that Realism could be a subset of Coherence. If we recall the definition of Realism (or Fidelity) by Alexander et al., it can be said to be comprised of three components: Physical Fidelity, Functional Fidelity, and Psychological Fidelity. Thus, logically, Coherence involves those aspects too.

Based on these definitions, we suggest Coherence (which encapsulates Realism), Immersion and Illusion as forming orthogonal axes, creating a comprehensive VR experience evaluation model (Figure 1). We can use this to describe the subjective feelings that arise in a user who encounters experiences placed along each axis within this space, including when supports are maximally provided. However, even though the given dimensions are orthogonal, and thus should not interact with each other, it is a challenge to measure the feeling even if we successfully provide a controlled experience. From a practical perspective, the orthogonal model could be represented using a Venn diagram approach, as can be seen in Figure 2. In this model, we also provide suggested or validated constructs for measuring the lower-level components. Based on the suggested Venn model, we argue that increased Realism can improve the chances of achieving feelings of deep Presence, including Co-presence and Social Presence, partially if the given Realism satisfied context, but not necessarily. Of course, measuring method should be considered but it is beyond our scope in this article.

\subsection{Implications}

Considering the proposed orthogonal model, Coherence that incorporates Realism does not directly enhance Illusion and Immersion. Improving sensory realism incurs a high cost due to the required hardware support. In this case, what is the motivation for providing realistic systems, such as multisensory-enabled platforms?

In our own work, we have repeatedly reported higher preference responses for our multisensory VR systems compared to typical VR systems, regardless of the context, number of sensory channels, and level of fidelity, even though multisensory cues did not consistently 


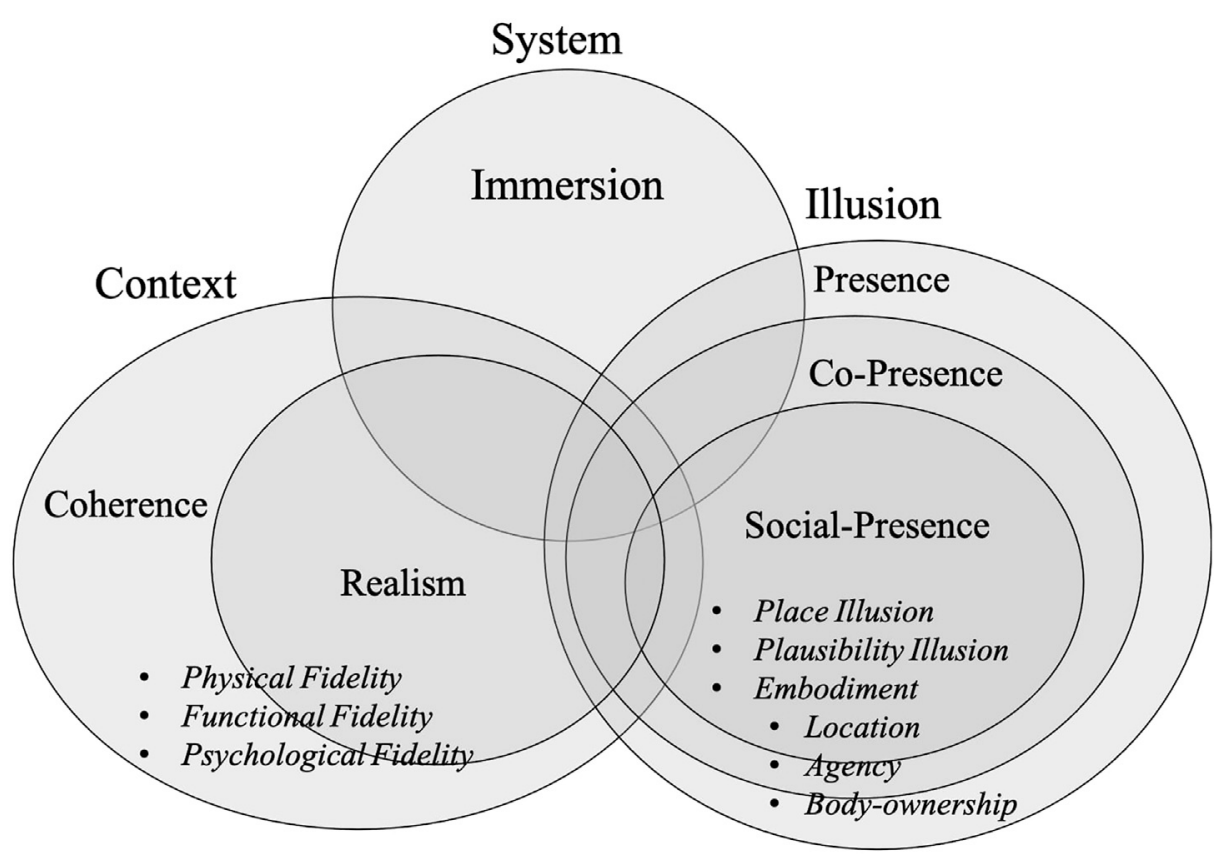

FIGURE 2 | Venn diagram model for quality of VR experience.

lead to higher Presence (Jung et al., 2020; Jung et al., 2021b; Jung et al., 2021a). The reasons are still not clear to us, but participants reported they felt a stronger sense of Fun, Impressiveness and Involvement in multisensory VR, which are strongly related to emotional engagement in the given place, object, and maybe certain events too, which sounds a lot like Aura (MacIntyre et al., 2004). Similarly, Doukakis et al. (2019) reported that visual feedback was the dominant factor chosen for designing VR on a limited budget. However, as the budget size increases, the preference for having a balanced distribution of resources (e.g., having additional smell feedback) increased. Thus, based on the observed and reported trends, we claim VR experiences with more and higher-quality stimuli fed to sensory systems might have stronger preference, and thus we suggest Preference as a new metric to evaluate VR experience as an exclusive factor from the proposed model. This is because the suggested model accounts for experiential attributes, but not other factors, such as cost, encumbrance, fun, or engagement. It is possible, for example, to have photorealistic graphics in gaming experiences that do not guarantee fun. The Nintendo Switch has a low-end system profile compared to the PlayStation or Xbox series in general, but user preference is similar among the systems. Thus we conclude that the proposed axis model works as a specific tool to evaluate the VR experience, along with three independent axes, and so we can evaluate solo VR experiences as well. On the other hand, Preference is a comparison tool that depends on the user's choice which might come from the overall experience compared to other given VR experiences. Most research does not tend to empathize the importance of User Preference in studies on VR experience. However, we argue that Preference can be a critical indicator in terms of business perspectives, since it clearly shows the overall evaluation from a direct comparison, and thus connects to people's preferences for future VR experiences.

\section{CONCLUSION}

In this article, we have reflected on two key high-level concepts, Immersion and Illusion. Based on previous work, we revisited the concepts of Coherence and Realism, and how they correlate with Illusion (as a representative of the sense of Presence) and Immersion. In order to explore these questions, we refer to a series of studies around the influence of multisensory cues in VR, and finally proposed two new models for representing the relationship between these three components, Coherence, Immersion, and Illusion. We conclude that these can be treated as independent dimensions, but that they might partially influence each other, as they intersect. Finally, we also suggest that researchers and designers consider Preference as a critical component for evaluating the impact of VR experiences, especially from a business perspective.

\section{DATA AVAILABILITY STATEMENT}

The original contributions presented in the study are included in the article/supplementary material, further inquiries can be directed to the corresponding author.

\section{AUTHOR CONTRIBUTION}

SJ and RL contributed to the ideation and development of this article. SJ developed the initial draft and RL contributed to further revision and polishing of the final article. 


\section{REFERENCES}

Alexander, A., Brunyé, T. T., Sidman, J., and Weil, S. A. (2005). From Gaming to Training: A Review of Studies on Fidelity, Immersion, Presence, and Buy-In and Their Effects on Transfer in Pc-Based Simulations and Games.

Banakou, D., Groten, R., and Slater, M. (2013). Illusory Ownership of a Virtual Child Body Causes Overestimation of Object Sizes and Implicit Attitude Changes. Proc. Natl. Acad. Sci. 110, 12846-12851. doi:10.1073/ pnas. 1306779110

Beckhaus, S., and Lindeman, R. W. (2011). Experiential Fidelity: Leveraging the Mind to Improve the VR Experience. Vienna: Springer Vienna, 39-49. doi:10.1007/978-3-211-99178-7_3

Bowman, D. A., and McMahan, R. P. (2007). Virtual Reality: How Much Immersion Is Enough? Computer 40, 36-43. doi:10.1109/MC.2007.257

Dinh, H. Q., Walker, N., Hodges, L. F., Chang Song, C., and Kobayashi, A. (1999). "Evaluating the Importance of Multi-Sensory Input on Memory and the Sense of Presence in Virtual Environments," in Proceedings IEEE Virtual Reality (Cat. No. 99CB36316), Houston, TX, USA, 13-17 March 1999, 222-228. doi:10.1109/ VR.1999.756955

Doukakis, E., Debattista, K., Bashford-Rogers, T., Dhokia, A., Asadipour, A., Chalmers, A., et al. (2019). Audio-visual-olfactory Resource Allocation for Tri-modal Virtual Environments. IEEE Trans. Visualization Comput. Graphics 25, 1865-1875. doi:10.1109/tvcg.2019.2898823

Feng, M., Dey, A., and Lindeman, R. W. (2016). “An Initial Exploration of a MultiSensory Design Space: Tactile Support for Walking in Immersive Virtual Environments," in 2016 IEEE Symposium on 3D User Interfaces (3DUI), Greenville, SC, USA, 19-20 March 2016, 95-104. doi:10.1109/ 3DUI.2016.7460037

Feng, M., Lindeman, R. W., Abdel-Moati, H., and Lindeman, J. C. (2015). "Haptic ChairIO: A System to Study the Effect of Wind and Floor Vibration Feedback on Spatial Orientation in VEs." In 2015 IEEE Symposium on 3D User Interfaces (3DUI), Arles, France, 23-24 March 2015, 149-150. doi:10.1109/ 3DUI.2015.7131744

Goffman, E. (1963). Behavior in Public Places; Notes on the Social Organization of Gatherings / Erving Goffman. New York: Free Press.

Hinckley, K., Pausch, R., Goble, J. C., and Kassell, N. F. (1994). "Passive Real-World Interface Props for Neurosurgical Visualization," in CHI '94 Proceedings of the SIGCHI Conference on Human Factors in Computing Systems, Boston, MA (New York, NY, USA: Association for Computing Machinery), 452-458. doi:10.1145/191666.191821

Insko, B. E. (2001). Passive Haptics Significantly Enhances Virtual Environments. Ph.D. thesis (Chapel Hill, NC: The University of North Carolina at Chapel Hill). AAI3007820.

Jung, S., and Hughes, C. E. (2016). "The Effects of Indirect Real Body Cues of Irrelevant Parts on Virtual Body Ownership and Presence," in ICAT-EGVE '16 Proceedings of the 26th International Conference on Artificial Reality and Telexistence and the 21st Eurographics Symposium on Virtual Environments, Little Rock, AR (Goslar, DEU: Eurographics Association), 107-114.

Jung, S., Karki, N., Slutter, M., and Lindeman, R. W. (2021a). On the Use of Multi-Sensory Cues in Symmetric and Asymmetric Shared Collaborative Virtual Spaces. Proc. ACM Hum.-Comput. Interact 5, 1-25. doi:10.1145/ 3449146

Jung, S., Li, R., McKee, R., Whitton, M. C., and Lindeman, R. W. (2021b). "Floor-vibration Vr: Mitigating Cybersickness Using Whole-Body Tactile Stimuli in Highly Realistic Vehicle Driving Experiences," in 2021 IEEE Conference on Virtual Reality and 3D User Interfaces (VR), Lisbon, Portugal.

Jung, S., Sandor, C., Wisniewski, P. J., and Hughes, C. E. (2017). "Realme: The Influence of Body and Hand Representations on Body Ownership and Presence," in SUI '17 Proceedings of the 5th Symposium on Spatial User Interaction, Brighton, United Kingdom (New York, NY, USA: Association for Computing Machinery), 3-11. doi:10.1145/3131277.3132186

Jung, S., Wisniewski, P. J., and Hughes, C. E. (2018). "In Limbo: The Effect of Gradual Visual Transition between Real and Virtual on Virtual Body Ownership Illusion and Presence," in 2018 IEEE Conference on Virtual Reality and 3D User Interfaces (VR), Tuebingen/Reutlingen, Germany, 1822 March 2018, 267-272. doi:10.1109/VR.2018.8447562
Jung, S., Wood, A. L., Hoermann, S., Abhayawardhana, P. L., and Lindeman, R. W. (2020). "The Impact of Multi-Sensory Stimuli on Confidence Levels for Perceptual-Cognitive Tasks in Vr," in 2020 IEEE Conference on Virtual Reality and 3D User Interfaces (VR), Atlanta, GA, USA, 22-26 March 2020, 463-472. doi:10.1109/VR46266.2020.00067

Kilteni, K., Maselli, A., Kording, K. P., and Slater, M. (2015). Over My Fake Body: Body Ownership Illusions for Studying the Multisensory Basis of Own-Body Perception. Front. Hum. Neurosci. 9, 141. doi:10.3389/fnhum.2015.00141

Latoschik, M. E., Roth, D., Gall, D., Achenbach, J., Waltemate, T., and Botsch, M. (2017). "The Effect of Avatar Realism in Immersive Social Virtual Realities," in Proceedings of the 23rd ACM Symposium on Virtual Reality Software and Technology.

Lindeman, R., Sibert, J., and Hahn, J. (1999). "Hand-held Windows: towards Effective 2d Interaction in Immersive Virtual Environments," in Proceedings IEEE Virtual Reality (Cat. No. 99CB36316), Houston, TX, USA, 13-17 March 1999, 205-212. doi:10.1109/VR.1999.756952

Lombard, M., Ditton, T., Crane, D., Davis, B., Gil-Egui, G., Horvath, K., et al. (2000). "Measuring Presence: A Literature-Based Approach to the Development of a Standardized Paper-And-Pencil Instrument," in Third International Workshop on Presence, Delft, Netherlands.

MacIntyre, B., Bolter, J., and Gandy, M. (2004). "Presence and the Aura of Meaningful Places," in 7th Annual International Workshop on Presence (PRESENCE 2004). Polytechnic University of Valencia: Valencia, Spain.

McDonnell, R., Breidt, M., and Bülthoff, H. H. (2012). Render Me Real? Investigating the Effect of Render Style on the Perception of Animated Virtual Humans. ACM Trans. Graph. 31, 1-11. doi:10.1145/2185520.2185587

Meehan, M., Insko, B., Whitton, M., and Brooks, F. P. (2002). Physiological Measures of Presence in Stressful Virtual Environments. ACM Trans. Graph. 21, 645-652. doi:10.1145/566654.566630

Milgram, P., and Kishino, F. (1994). A Taxonomy of Mixed Reality Visual Displays. IEICE Trans. Inf. Syst. 77, 1321-1329.

Naef, M., Staadt, O., and Gross, M. (2002). "Spatialized Audio Rendering for Immersive Virtual Environments," in VRST '02 Proceedings of the ACM Symposium on Virtual Reality Software and Technology, Hong Kong, China (New York, NY, USA: Association for Computing Machinery), 65-72. doi:10.1145/585740.585752

Nowak, K. L., and Biocca, F. (2003). The Effect of the agency and Anthropomorphism of Users' Sense of Telepresence, Copresence, and Social Presence in Virtual Environments. Presence: Teleoper. Virtual Environ. 12, 481-494. doi:10.1162/105474603322761289

Riccio, G. E. (1995). "Coordination of Postural Control and Vehicular Control: Implications for Multimodal Perception and Simulation of Self-Motion," in Local Applications of the Ecological Approach to Human-Machine Systems. Editor J. C. K. V. Peter Hancock (John Flach), 60.

Samad, M., Chung, A. J., and Shams, L. (2015). Perception of Body Ownership Is Driven by Bayesian Sensory Inference. PLOS ONE 10, 1-23. doi:10.1371/ journal.pone.0117178

Schroeder, R. (2005). "Copresence and Interaction in Virtual Environments: An Overview of the Range of Issues," in Presence 2002: Fifth International Workshop, Porto, Portugal, 274-295.

Skarbez, R., Brooks, F. P., Jr., and Whitton, M. C. (2017). A Survey of Presence and Related Concepts. ACM Comput. Surv. 50, 1-39. doi:10.1145/3134301

Skarbez, R. (2016). Plausibility Illusion in Virtual Environments. Chapel Hill, NC: University of North Carolina at Chapel Hill. doi:10.17615/2mg3-gh93

Skarbez, R., Smith, M., and Whitton, M. C. (2021). Revisiting Milgram and Kishino's Reality-Virtuality Continuum. Front. Virtual Reality 2, 27. doi:10.3389/frvir.2a021.64799710.3389/frvir.2021.647997

Slater, M. (1999). Measuring Presence: A Response to the Witmer and Singer Presence Questionnaire. Presence: Teleoper. Virtual Environ. 8, 560-565. doi:10.1162/105474699566477

Slater, M. (2009). Place Illusion and Plausibility Can lead to Realistic Behaviour in Immersive Virtual Environments. Philos. Trans. R. Soc. B: Biol. Sci. 364, 3549-3557. doi:10.1098/rstb.2009.0138

Slater, M., Spanlang, B., and Corominas, D. (2010). Simulating Virtual Environments within Virtual Environments as the Basis for a Psychophysics of Presence. ACM Trans. Graph. 29, 1-9. doi:10.1145/1778765.1778829

Stoffregen, T. A., Bardy, B. G., Smart, L. J., and Pagulayan, R. J. (2003). "On the Nature and Evaluation of Fidelity in Virtual Environments: Applications, 
Implications, and Human Performance," in Virtual and Adaptive Environments. Editors L. J. Hettinger and M. W. Haas (Mahwah, NJ: Lawrence Erlbaum Associates, Inc), 111-127.

Waltemate, T., Gall, D., Roth, D., Botsch, M., and Latoschik, M. E. (2018). The Impact of Avatar Personalization and Immersion on Virtual Body Ownership, Presence, and Emotional Response. IEEE Trans. Visualization Comput. Graphics 24, 1643-1652. doi:10.1109/TVCG.2018.2794629

Witmer, B. G., and Singer, M. J. (1998). Measuring Presence in Virtual Environments: A Presence Questionnaire. Presence: Teleoperators and Virtual Environments 7, 225-240. doi:10.1162/ 105474698565686

Yuan, Y., and Steed, A. (2010). "Is the Rubber Hand Illusion Induced by Immersive Virtual Reality?,” in 2010 IEEE Virtual Reality Conference (VR),
Boston, MA, USA, 20-24 March 2010, 95-102. doi:10.1109/ VR.2010.5444807

Conflict of Interest: The authors declare that the research was conducted in the absence of any commercial or financial relationships that could be construed as a potential conflict of interest.

Copyright (c) 2021 Jung and Lindeman. This is an open-access article distributed under the terms of the Creative Commons Attribution License (CC BY). The use, distribution or reproduction in other forums is permitted, provided the original author(s) and the copyright owner(s) are credited and that the original publication in this journal is cited, in accordance with accepted academic practice. No use, distribution or reproduction is permitted which does not comply with these terms. 